\title{
Transdermal Delivery of Active Principles with Pulsed RF Contact Diathermy - A Narrative Review
}

\author{
Michele Gallamini $^{*}$, Gianluca Bernabei ${ }^{2}$, Eugenio Pecchioli ${ }^{3}$ \\ ${ }^{1}$ Eng. PhD Medical Devices Freelance Researcher https://orcid.org/0000-0002-4550-526X Sal. \\ Maggiolo di Nervi, 716167 Genoa, Italy (MG) \\ ${ }^{2}$ FKT Fisiocrea Srl Baldissero Canavese, Turin, Italy https://fisiohealing.com/ (GB). \\ ${ }^{3}$ MD Surgery Specialist, Omotoxicology expert, Via Masaccio, 235, 50132 Florence; Italy drpecchioli@tin.it (EP) \\ *Corresponding author: Michele Gallamini; michele.gallamini@fastwebnet.it
}

Received 18 February 2021;

Accepted 06 March 2021;

Published 17 March 2021

\begin{abstract}
The transdermal delivery of drugs and, in more general terms, of active principles can be performed by iontophoresis and/or by electroporation. The advantages of a local treatment vs. other drugs intake methods are well known as it reduces the systemic involvement and therefore the systemic contraindications. To perform the treatment exploiting the electroporation effect there is nowadays a specific variant of a known medical device used in physiotherapy: the Medium Frequency Resistive/Capacitive contact Diathermy. The adoption of a power control exploiting Pulse Width Modulation of the applied current adds to the device also this capability of great potential in rehabilitation and aesthetic medicine treatments. The theoretic approach is discussed and applications suggestions are being given.
\end{abstract}

Keywords: MF contact Diathermy, Pulse Width Modulation, Transdermal drug delivery, Electroporation, RF electroporation

\section{Introduction}

Medium Frequency Contact Diathermy (0.5 to $1 \mathrm{MHz})$ is increasingly used in Physical Therapy as well as in Aesthetical Medicine. It consists in the application of an alternate current with voltages in excess of a few hundred Volts at frequencies ranging from 0.5 to some $1 \mathrm{MHz}$ through contact electrodes one of which can be either conductive (for a "resistive" application) or nonconductive (for a "capacitive" treatment). While in the "resistive" mode the main effects are depending on the current flow, in the "capacitive" mode the main effects are depending on the applied voltage. The return electrode, common to both modes, is a conductive panel applied on the patient's skin according to a geometry that ensures the flow of the current through the tissues that need the specific stimulation. Born at the turn of the 20th century from the studies of N. Tesla ${ }^{[1]}$ and J.A. D'Arsonval ${ }^{[2]}$ this kind of therapy was defined diathermy by C.F. Nagelschmidt in 1906 because it was observed that the main effects of the alternate current flow through human tissues were at first a neuromuscular stimulation disappearing at frequencies of a few hundred $\mathrm{Hz}$, then re-appearing at frequencies around $0.5 \mathrm{MHz}$ with a significant raise of temperature in the inner part of the ex-posed tissues. Around 1930 preference was given to shorter waves (range $20-30 \mathrm{MHz})^{[3]}$ and in 1973 the U.S. Food and Drugs Administration decided to recognize both
- $\quad$ A Short-Wave Diathermy (27.1 MHz)

- A Microwave Diathermy working either at $915 \mathrm{MHz}$ or at $2450 \mathrm{MHz}$.

In spite of that, at the end of the 20th century the technique, described also by $\mathrm{H}$ Hesse ${ }^{[4]}$ that underwent successfully to the treatment of low-back-pain by that technique in 1924 at BadenBaden, reappeared in a patented upgraded model by J. Calbet ${ }^{[5]}$ of INDIBA SA (Barcelona). The device has been gaining the increasing favour of Physiotherapists to the point that in Italy alone there is an estimated population of more than 20.000 units, known under the generic name of TECAR (it stands for the Italian Trasferimento Energetico CApacitivo e Resistivo, in English CRET for Capacitive and Resistive Energy Transfer) of several make. Common features are the working frequencies (in the range from $200 \mathrm{KHz}$ to $2 \mathrm{MHz}$ ), the application modes between two contact electrodes one of which can be either conductive (Resistive mode) or insulated (capacitive mode) and the power ranging up to some $300 \mathrm{~W}$. Power is generally controlled by regulating the applied stimulation voltage under the assumption that the key beneficial effects of MF Diathermy are depending on the thermal effects produced by the current flow through the tissues. Among Physiotherapists the TECAR application is generally defined as athermic (for a low level of applied power that is not inducing perceivable heat), omo-thermic (for medium level applied power felt at the same temperature of the patients' body), thermic (for a 
medium-to-high level of applied power inducing a perceivable heat level felt comfortable and pleasant by the patient), hyper-thermic (for a high level of applied power that the patient perceives as almost unbearably hot). The application is performed by applying the current through the electrodes in contact with the skin. A conductive cream is used to ensure a steady homogeneous contact while the electrode is moved by the therapist along the area to be treated.

Among the effects of time-varying electric voltage applications to biologic tissues a peculiar one, consisting in the formation of pores across the lipidic layer under the skin when stimuli in the range 100 thru $1500 \mathrm{~V}$ decaying in 1 thru 10,000 $\mu$ sec were applied. By the term "Electroporation" ${ }^{[6]}$ it was defined that temporary perturbation of the twin layer lipidic membrane and rather quickly in 1993 its exploitation for topical delivery of active principles was proposed ${ }^{[7]}$.

\section{Transdermal active delivery principles}

The transdermal delivery of active principles can offer significant advantages in front of traditional assumption methods involving either the gastrointestinal tracts (oral or rectal) or the vascular system (intramuscular or intravenous infiltration) or else the respiratory ducts (sprays, aerosols, etc) because it can reach a specific target with marginal involvement of systemic metabolic activities ${ }^{[8,9]}$.

In spite of its advantages, it is not so easy to perform the transdermal delivery. The most known, although discussed, is the Mesotherapy, defined as follows: "Mesotherapy involves the use of multiple intradermal or subcutaneous injections of a mixture of compounds in minute doses, by means of very fine needles, directly over/near the affected sites" ${ }^{[10]}$. It is by all evidence a discrete distribution, by definition inhomogeneous both in space and in time.

A different approach can be sought with electrical assistance by:

- Promoting the permeability of the skin through the formation of pores on the twin layer lipidic membrane (Poration) ${ }^{[11]}$, or

- Pushing active principles molecules through the skin (iontophoresis) $^{[12]}$

While the first one is mechanically opening channels through which molecules can be absorbed, the second one requires the molecule to be electrically polarized in order to be displaced by the generally tiny electric field (a voltage under $10 \mathrm{~V}$ is generally required).

\section{Electroporation}

Poration can be electrically promoted by electrical stimulation that, probably by polarizing lipidic polymers open aqueous channels ${ }^{[13]}$ where molecules, even large ones, either electrically loaded or inert, can pass through with a surprisingly quick effect ${ }^{[14]}$ that do suggest a remarkable potential of application in traumatology.

Among the active principles that were tested in electroporation there are also compounds with a molecular weight between 18 and 38,000 Dalton. Worth signalling TETRACAINE (local anaesthetic) ${ }^{[15]}$, FENTANYL [16,17], NONIVAMIDE, Salmon CALCITONIN ${ }^{[18,19]}$, DEXTRAN SULPHATE ${ }^{[20,21]}$, HEPARIN ${ }^{[22]}, \quad \mathrm{C}$ VITA-MIN ${ }^{[23]}$ LIDOCAINE ${ }^{[24]}$, OLIGONUCLEOTIDES ${ }^{[25]}$.

More recent publications dealing with evidences in oncologic applications [26,27] suggest the wide field of investigations opening up to the research along this specific line. The transdermal behaviour appears to be affected by the intensity, the duration, the shape and the repetition frequency of the electric pulses (see Figure 1). In general terms a key factor is the pulse width that should be consistently in the order of magnitude of 1 $\mu \sec ^{[28,29]}$.

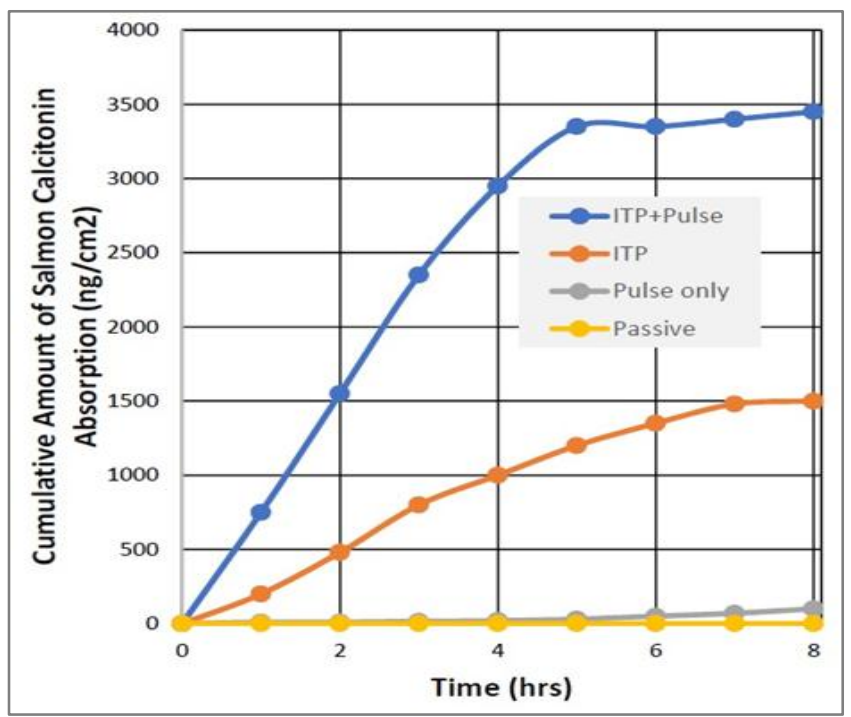

Figure 1

Salmon Calcitonin absorption through the human skin

a) in normal diffusion

b) in $4 \mathrm{~h}$ iontophoresis at $0,5 \mathrm{~mA} / \mathrm{cm}^{2}$

c) with $6 \times 120 \mathrm{~V}$ pulses lasting $10 \mathrm{~ms}$ and iontophoresis as above from Chang ${ }^{[18]}$

Radiofrequency electroporation even devoid of thermal effects has been demonstrated ${ }^{[30]}$. However, some comments, more specific with regard to the MF radiofrequency, should be added.

MF Diathermy exposes the patient's tissues to the stimulation with an alternate current at high voltage (Vpp $1000 \mathrm{~V}$ ) and frequencies between 100 and $1,000 \mathrm{KHz}$ (Wave period between 20 and $2 \mu \mathrm{sec}$ ) with an half-wave duration ranging from 10 to $1 \mu$ sec similar to the typical diadynamic pulse used to electrically create pores in the human skin (see Figure 2).

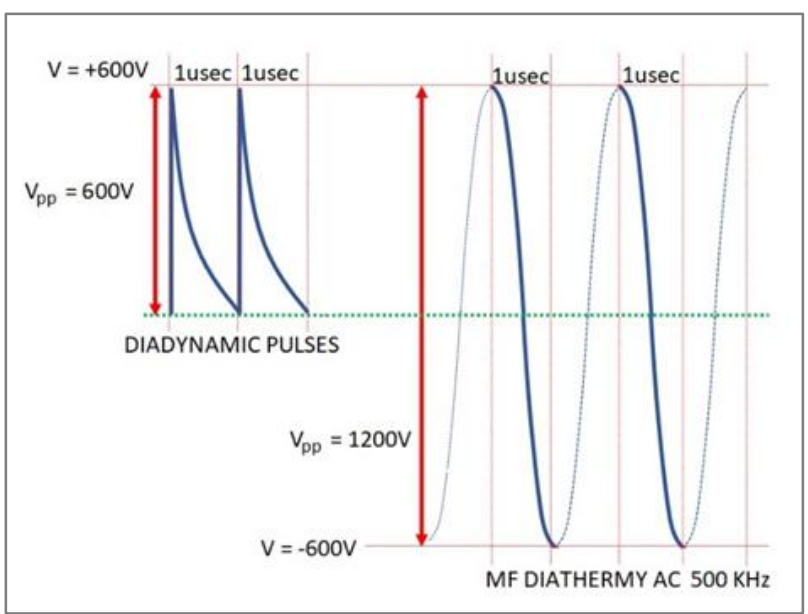

Figure 2: Diadinamic pulses

To start the phenomenon a voltage of at least $200 \mathrm{Vpp}$ has to be applied.

The majority of MF Diathermy devices nowadays available on the market do control the output power in the most traditional way: i.e., through the output voltage control. The higher the voltage, the higher the power to the patient's tissues. It can be 
reasonably expected that to achieve the required voltage an output power of some $150 \mathrm{~W}$ should be applied.

On the other hand, the diadynamic pulses should be applied in bursts of a few ones and not in a continuous mode. This is an intrinsic feature for MF Diathermy devices whose power is modulated with Pulse Width Modulation (PWM).

The concept, widely applied in many electrically operated systems, is basically consisting in the rhythmical On-Off switching of the applied current and regulate the average current applied to the mechanism through the variation of the emission duration. Under these conditions the mean applied power is proportional to the ratio between the active time and the intervals between the pulses that is normally referred to as Duty Cycle (See Figure 3). Duty Cycle that can vary between 0 and 1 is generally expressed in $\%$ value.

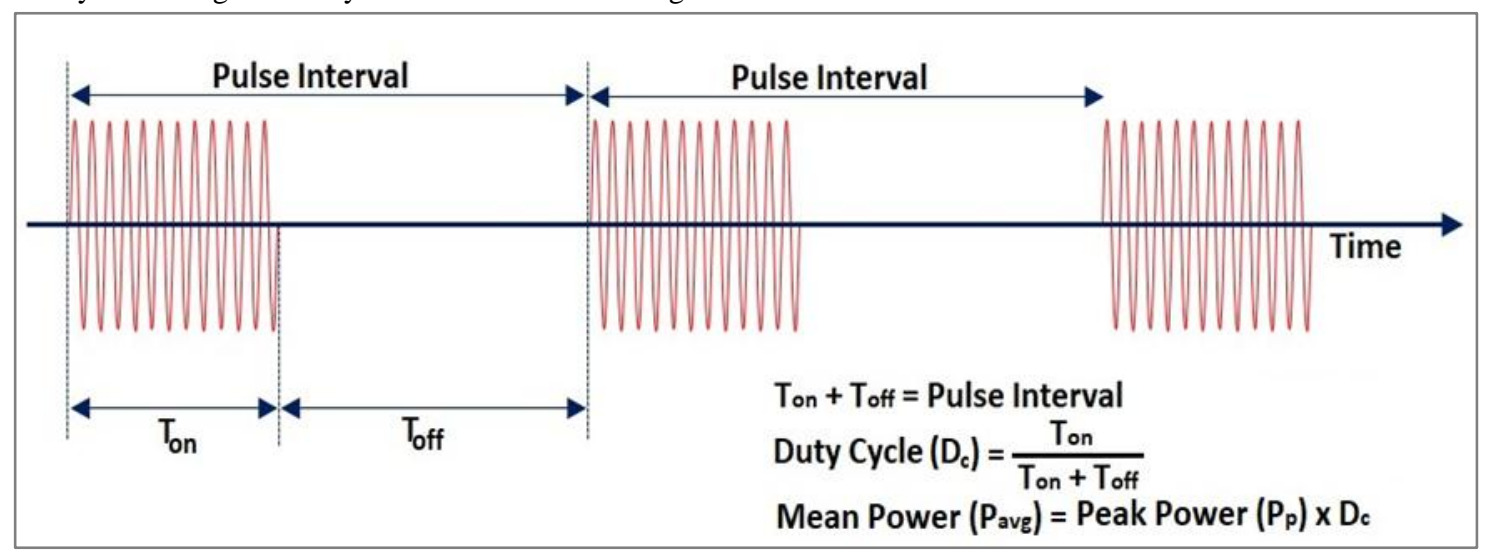

Figure 3: Pulse Width Power Mpdulation

The main advantage of PWM in Diathermy is the possibility to exploit the stimulating effects of the applied voltage selecting the quantity of energy as appropriate to the pathology.

With a classical Voltage control, the Voltage value required for electroporation will probably correspond to a level of power too high to be applied to a patient in acute or inflamed conditions. In fact, the instantaneous absorbed energy is proportional to the applied voltage according to the law (Equation

$$
E=P \times t=\frac{V^{2}}{Z} \times t
$$

Where E stands for energy, $\mathrm{P}$ stands for power, $\mathrm{t}$ stands for time, $\mathrm{V}$ stands for applied voltage, and Z, a vector, stands for impedance. That energy is converted to heat that, in turn, promotes vasodilation effects that might be undesirable under - for example - acute inflamed conditions. (See Figure 4). 1)

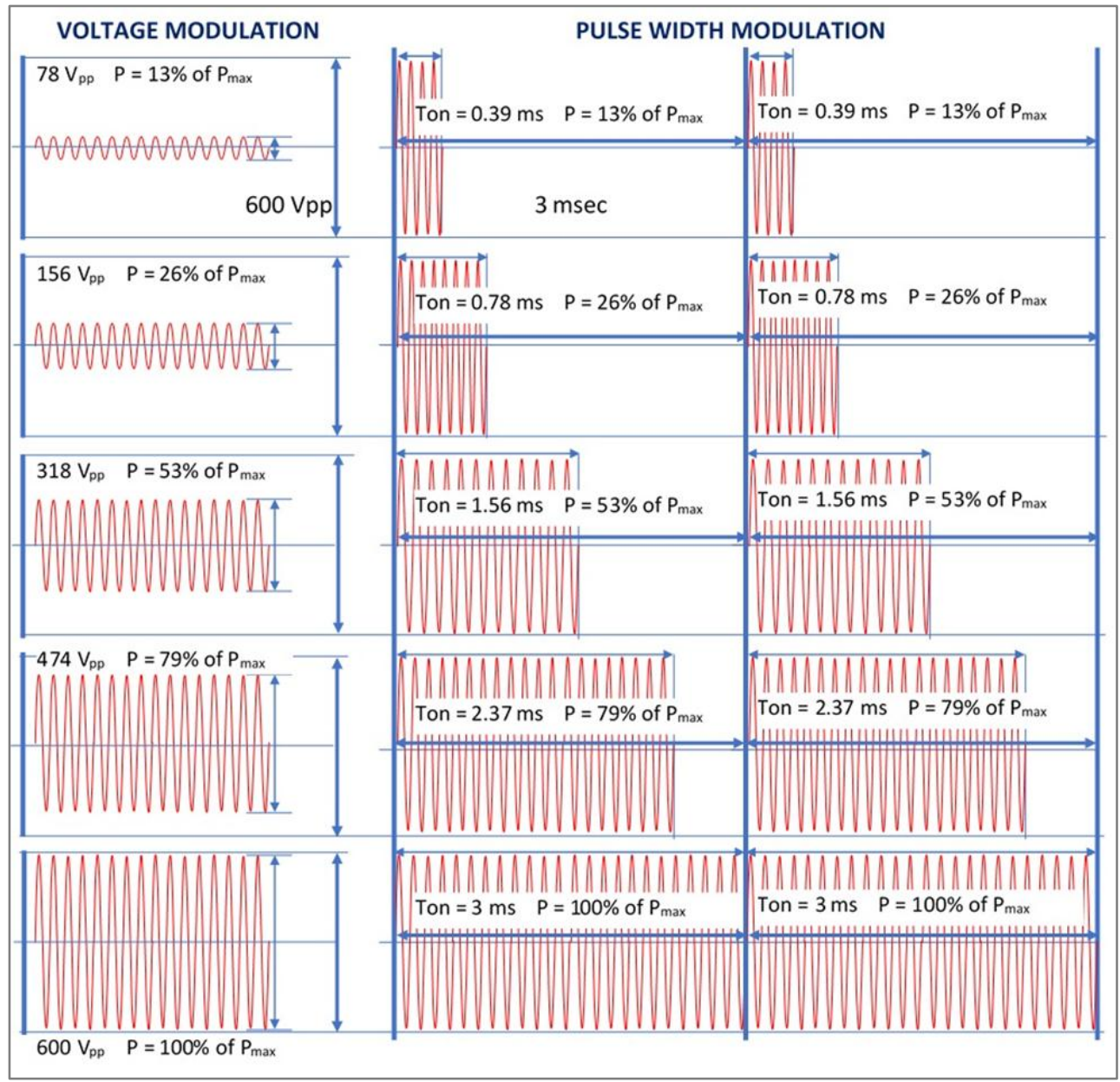

Figure 4: Voltage modulation vs pulse width modulation 
Besides the advantage of having the same Voltage at any selected mean power level thus exploiting the best voltage related effects of the stimulation (to be in the range $50-100 \mathrm{~V} / \mathrm{cm}$ between the two electrodes) ${ }^{[31]}$, the known effects of the pulsed stimulation resulting from the pulse repetition frequency of the PWM control are also available.

As said Diathermy can be applied either through one insulated electrode (Capacitive Mode) or through a conductive electrode (Resistive Mode). While in the Resistive Mode the main effect is bound to the current flow through the tissues with higher resistance such as bones, tendons and ligaments, in the Capacitive Mode the main effect is the ionic movement in the less resistive tissues and especially under the insulated contact electrode where tissue become the second armature of a capacitor (see Figure 5).

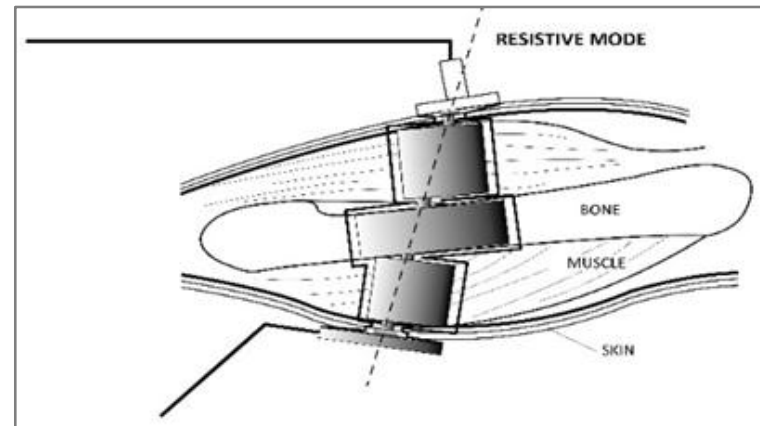

AVERAGE ABSORBED ENERGY - CONCEPTUAL

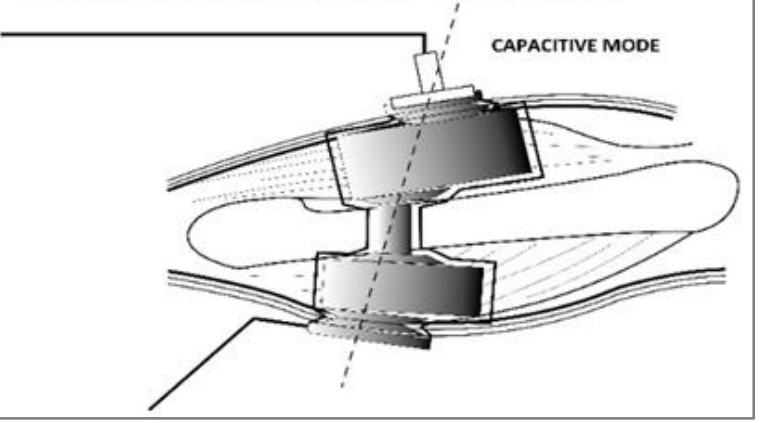

Figure 5: The energy transfer to tissues

The darker area is the one where energy concentrates in the specific application mode
The Capacitive Mode is affording a stimulating effect that is promoting a local vasoactive effect that promotes the absorption of the delivered active principle.

The particular range of frequency chosen for MF contact Diathermy is affording a further advantage: as demonstrated, for example, by the body mass bioimpedance analysers ${ }^{[32]}$, while under $500 \mathrm{KHz}$ the cell membrane impedance prevents the current flow in the intracellular space, above that frequency the current flows also within the intracellular spaces. Therefore, by choosing the treatment frequency it is possible, to some extent, to target the delivery either to the tissues' cells or to the extracellular space.

Although also drugs could be locally delivered by MF PWM Diathermy it is to remark that only drugs approved for local treatment in mesotherapy should be considered, because of the specific pharmaco-dynamics involved. A sound clinical indication can be derived from "Table 1 - Daily dose ranges and physicalchemical and pharmacokinetic properties of currently approved transdermal delivered drugs" of an already mentioned brilliant review by Wiedersberg and Guy (2014) ${ }^{[9]}$.

It is however to underline that while the "classical" MF Diathermy, heating the tissues, should not be applied in acute conditions or on inflamed tissues, the PWM based MF Diathermy could be applied and, by exploiting its characteristics, even at negligible mean power levels could be used for transdermal delivery of antalgic and myorelaxant drugs for an effective pain killing action.

Less critical appears the use of active principles not officially classified as drugs.

A specific reference should be made to several active principles that could be mixed with the conductive cream for local application to enhance the physiotherapy treatment ${ }^{[33,34]}$.

Among them there are all the herbal principles such as Arnica (Arnica Montana), Aloe (Aloe barbadensis Mille), Aescin, Allantoin (Consolida major Symphytum officinale), Horsetail (Equisetum arvense), Mallow (Malva sylvestris), Bergamot (Citrus bergamia), Cedar wood (Cedrus atlantica), Juniper (Juniperus communis), Lavender (Lavandula hybrida), as well as some chemicals as ETDA (etilendiamminotetraacetic acid) and the proposed mix known as Bach or Australian Flowers.

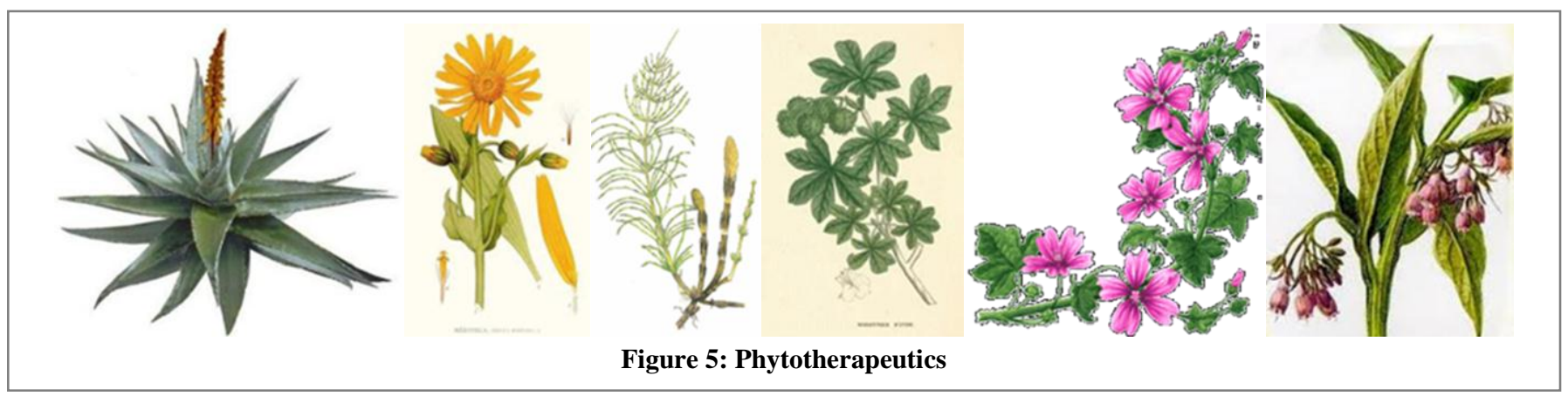

Homeopathic and Homotoxicology remedies can be as well administered through this method enjoying the benefit of a topical application

Worth mentioning that nowadays, besides the diluted active principles, there are available also MF Diathermy creams embodying also active principles to be applied on specific conditions.

\section{Multifrequency MF PWM Diathermy Electroporation - Application guidelines}

In general terms, the following criteria should be followed:
1) The "return" electrode should be positioned to concentrate the mesotherapy effect on the desired target: the distance between active and return electrode should be reduced as far as possible avoiding, whenever feasible, to involve between the electrodes lymphnode stations that could drain away the principle;

2) A thin layer of conductive cream should be laid on the skin both on the area where the return electrode is applied and, on the area where the active, manual, electrode will be made slide during the treatment; 
3) Add some drops of the hydro soluble active principle over the area on the thin layer of cream;

4) Apply diathermy at low or very low power $(<20 \mathrm{~W})$ in Capacitive mode to exploit the ionic transport for 5-10 min sliding the active electrode over the skin to promote the local absorption: the treatment will effectively cause the opening of the skin layer pores and the natural diffusion of the principle under the skin;

5) Increase slowly the applied power up to $100 \mathrm{~W}$ sliding the electrode to promote the local absorption of the active principle as the increase of temperature - always within limits pleasant to the patient - will promote the local vasodilation. Worth stressing the fact that vasodilation should be avoided in acute conditions although biostimulation effects are not dependent on temperature increase that should be avoided in acute conditions: in those conditions, longer lasting applications at lower power levels might be recommended.

The choice of the applied frequency, whenever applicable, offers a specific opportunity: a frequency under $500 \mathrm{KHz}$ would promote the diffusion of the principle primarily in the Extracellular Soft Tissue Matrix while a higher frequency might involve also the cells.

\section{Conclusions}

MF Diathermy can be used to promote absorption of active principles by the treated tissues. The Diathermy emission power should be controlled through PWM technique in order to exploit the highest de-vice Voltage capable to promote the pores opening even at very low mean power setting and with the possibility of at least two carrier frequencies across the $500 \mathrm{KHz}$ value to afford both the capability of extracellular and intracellular diffusion of the active principles. Studies to deepen the understanding of specific pharmacodynamics can open up further mesotherapy opportunities.

\section{Conflicts of Interest}

Michele Gallamini is a minority shareholder $(<1 \%)$ of Fremslife Srl producing a MF Diathermy device. Gianluca Bernabei and Eugenio Pecchioli declare that there is no conflict of interest regarding the publication of this paper.

\section{Funding Statement}

No funding was received

\section{Authors' contributions}

MG conceived and drafted the manuscript and the figures. GB expert user of MF Diathermy devices in Rehabilitation was providing inputs both in the general conception of the paper and in the definition of the application guidelines. EP, expert on omotoxicology, and specialist in the application of MF diathermy on aesthetic medicine gave his specific contribution. All authors read and approved the final manuscript.

\section{References}

[1] Nikola Tesla (10 July 1856 - 7 January 1943) was a Serbian-American inventor, electrical engineer, mechanical engineer, and futurist who is best known for his contributions to the design of the modern alternating current (AC) electric-ity supply system. (Wikipedia)

[2] Jacques-Arsène d'Arsonval (June 8, 1851 - December 31, 1940) was a French physician, an important contributor to the emerging field of electrophysiology, the study of the effects of electricity on biological organisms, in the nineteenth century. (Wikipedia)

[3] Geddes LA. A short history of the electrical stimulation of excitable tissue. Including electrotherapeutic applications. Physiologist. 1984 Feb;27(1 Suppl):S1-47

[4] Kurgast. Aufzeichnungen von einer Badener Kur, 1925

[5] Calbet J. Tratado de la transferencia eléctrica capacitive [Spanish]. Barcelona Doyma. 1992

[6] AR Denet, R Vanveber, V Preat Skin electroporation for transdermal and topical delivery. Adv Drug Deliv Rev. 2004 Mar 27;56(5):659-74. Review

[7] Prausnitz MR, Bose VG, Langer R, Weaver JC. Electroporation of mammalian skin: a mechanism to enhance transdermal drug delivery. Proc Natl Acad Sci U S A. 1993 Nov 15;90(22):10504-8

[8] Heller R, Gilbert R, Jaroszeski MJ. Clinical applications of electrochemotherapy. Adv Drug Deliv Rev. 1999 Jan 4;35(1):119-129

[9] Wiedersberg S, Guy RH. Transdermal drug delivery: 30+ years of war and still fighting! J Control Re-lease. 2014 Sep 28;190:150-6

[10] Sivagnanam G. Mesotherapy - The french connection. J Pharmacol Pharmacother. 2010;1(1):4-8. doi:10.4103/0976-500X.64529

[11] Cevc G. Drug delivery across the skin. Expert Opin Investig Drugs. 1997 Dec;6(12):1887-937

[12] Guy, Richard H.; Kalia, Yogeshvar N.; Delgado-Charro, M.Begoña; Merino, Virginia; López, Alicia; Marro, Diego (2000). Iontophoresis: electrorepulsion and electroosmosis. Journal of Controlled Release. 64 (1-3): 129-132.

[13] Weaver JC, Vaughan TE, Chizmadzhev Y. Theory of electrical creation of aqueous pathways across skin transport barriers. Adv Drug Deliv Rev. 1999 Jan 4;35(1):21-39

[14] Vanbever R, Langers G, Montmayeur S, Préat V. Transdermal delivery of fentanyl: rapid onset of analgesia using skin electroporation. J Control Release. 1998 Jan 2;50(1-3):225-35

[15] Hu Q, Liang W, Bao J, Ping Q. Enhanced transdermal delivery of tetracaine by electroporation. Int $\mathrm{J}$ Pharm. 2000 Jul 20;202(1-2):121-4

[16] Vanbever R, LeBoulengé E, Préat V. Transdermal delivery of fentanyl by electroporation. I. Influence of electrical factors. Pharm Res. 1996 Apr;13(4):559-65

[17] Vanbever R, Morre ND, Préat V. Transdermal delivery of fentanyl by electroporation. II. Mechanisms involved in drug transport. Pharm Res. 1996 Sep;13(9):1360-6

[18] Chang SL, Hofmann GA, Zhang L, Deftos LJ, Banga AK. Transdermal iontophoretic delivery of salmon calcitonin. Int J Pharm. 2000 Apr 25;200(1):107-13

[19] Chang SL, Hofmann GA, Zhang L, Deftos LJ, Banga AK. The effect of electroporation on iontopho-retic transdermal delivery of calcium regulating hormones. J Control Release. 2000 May 15;66(2-3):127-33

[20] Of great interest for aesthetic medicine (rosacea, antiaging) 
[21] Badkar AV, Banga AK. Electrically enhanced transdermal delivery of a macromolecule. J Pharm Pharmacol. 2002 Jul;54(7):907-12

[22] Prausnitz MR, Edelman ER, Gimm JA, Langer R, Weaver JC. Transdermal delivery of heparin by skin electroporation. Biotechnology (N Y). 1995 Nov;13(11):1205-9

[23] Zhang L, Lerner S, Rustrum WV, Hofmann GA. Electroporation-mediated topical delivery of vita-min C for cosmetic applications. Bioelectrochem Bioenerg. 1999 May;48(2):453-61

[24] Wallace MS, Ridgeway B, Jun E, Schulteis G, Rabussay $\mathrm{D}$, Zhang L. Topical delivery of lidocaine in healthy volunteers by electroporation, electroincorporation, or iontophoresis: an evaluation of skin anesthesia. Reg Anesth Pain Med. 2001 May-Jun;26(3):229-38

[25] Regnier V, De Morre N, Jadoul A, Préat V. Mechanisms of a phosphorothioate oligonucleotide de-livery by skin electroporation. Int J Pharm. 1999 Jul 20;184(2):147-56

[26] Hojman P, Gissel H, Andre FM, Cournil-Henrionnet C, Eriksen J, Gehl J, Mir LM. Physiological effects of high and low-voltage pulse combinations for gene electrotransfer in muscle. Hum Gene Ther. 2008 Nov;19(11):1249-60

[27] André FM, Gehl J, Sersa G, Préat V, Hojman P, Eriksen J, Golzio M, Cemazar M, Pavselj N, Rols MP, Miklavcic D, Neumann E, Teissié J, Mir LM. Efficiency of high and low-voltage pulse combinations for gene electrotransfer in muscle, liver, tumor, and skin. Hum Gene Ther. 2008 Nov;19(11):1261-71

[28] Lee JW, Gadiraju P, Park JH, Allen MG, Prausnitz MR. Microsecond thermal ablation of skin for transdermal drug delivery. J Control Release. 2011 Aug 25;154(1):58-68

[29] Denet AR, Vanbever R, Préat V. Skin electroporation for transdermal and topical delivery. Adv Drug Deliv Rev. 2004 Mar 27;56(5):659-74. Review.

[30] Sintov AC, Krymberk I, Daniel D, Hannan T, Sohn Z, Levin G. Radiofrequency-driven skin micro-channeling as a new way for electrically assisted transdermal delivery of hydrophilic drugs. J Con-trol Release. 2003 Apr 29;89(2):311-20

[31] M. Y. Sulaeman, R. Widita The effect of electric field intensity, pulse width, and pulse rise time on conventional and supra electroporation THE 5TH INTERNATIONAL CONFERENCE ON MATHEMATICS AND NATURAL SCIENCES AIP Conference Proceedings Volume 1677, Issue 1 DOI: 10.1063/1.4930766

[32] Jaffrin MY, Morel H. Body fluid volumes measurements by impedance: A review of bioimpedance spectroscopy (BIS) and bioimpedance analysis (BIA) methods. Med Eng Phys. 2008 Dec;30(10):1257-69

[33] Bernabei G. - Pecchioli E. Applicazioni pratiche dell'utilizzo della diatermia nelle patologie della spalla Inquadramento olistico (Holistic approach to the practical application of Diathermy to shoulder pathologies) [Italian] Ed. Martina Bologna Italy ISBN 978-88-7572067-4

[34] Bernabei G. - Pecchioli E. Applicazioni pratiche dell'utilizzo della diatermia nelle patologie del ginocchio - Inquadramento olistico (Holistic approach to the practical application of Diathermy to knee pathologies) Ed. Martina Bologna Italy ISBN 978-88-7572-091-9 